\title{
Ultrafast Electron Localization Dynamics Following Photo-Induced Charge Transfer
}

\author{
Alvin T. Yeh, ${ }^{1,2}$ Charles V. Shank, ${ }^{1,2}$ James K. McCusker ${ }^{1 *}$
}

Molecular dynamics occurring in the earliest stages following photo-induced charge transfer were investigated. Femtosecond time-resolved absorption anisotropy measurements on $\left[\mathrm{Ru}(\mathrm{bpy})_{3}\right]^{2+}$, where bpy is $2,2^{\prime}$-bipyridine, reveal a time dependence in nitrile solutions attributed to initial delocalization of the excited state over all three ligands followed by charge localization onto a single ligand. The localization process is proposed to be coupled to nondiffusive solvation dynamics. In contrast, measurements sampling population dynamics show spectral evolution associated with wave packet motion on the excited state surface that is independent of solvent. The results therefore reveal two important contributions to the evolution of charge transfer states in condensed phase, one that is strongly coupled to the surrounding environment and another that follows a potential internal to the molecule.

Photo-induced charge transfer processes are central to a wide range of important physical and chemical phenomena. Perhaps best known is the photo-induced charge separation that occurs in photosynthetic reaction centers, yielding a transmembrane potential gradient that ultimately drives the production of adenosine triphosphate (1). In materials science, light absorption by wide band gap semiconductors results in the formation of conduction band electrons that are the basis for photoelectron conversion in photovoltaic devices (2). The surrounding medium can be an important factor in directing the course of chemical dynamics in condensed phase. When the dynamics of the chemical process in question are slow relative to the response of the medium, a quasi-steady-state condition exists between the chromophore and its environment, and the overall effect of the solvent is largely thermodynamic. Recently, there has been considerable interest both experimentally and theoretically in the dynamics of solvation, that is, when the chemistry and medium response to that chemistry occur on comparable time scales (3-8). Under these circumstances, the role of solvent dynamics in the kinetics and mechanism of excited-state evolution can be profound. Transition metal complexes in particular can exhibit charge transfer transitions between the metal center and ligand(s) bound to that metal, a process that necessarily involves charge redistribution on the periphery of the chromophore. Because the ligands are interacting with the

'Department of Chemistry, University of California, Berkeley, CA 94720, USA. ${ }^{2}$ Materials Sciences Division, Lawrence Berkeley National Laboratory, Berkeley, CA 94720, USA.

*To whom correspondence should be addressed. Email: mccusker@socrates.berkeley.edu solvent, such compounds provide a convenient platform for examining how solvation dynamics couple to photo-induced charge transfer events.

Tris-(2,2'-bipyridine)ruthenium(II), abbreviated $\left[\mathrm{Ru}(\mathrm{bpy})_{3}\right]^{2+}$, is the prototype for a large class of chromophores whose study has formed the basis for most of the transition metal-based solar energy conversion schemes of the past two decades $(9,10)$. $\left[\mathrm{Ru}(\mathrm{bpy})_{3}\right]^{2+}$ has been extensively studied, and much is known about the properties of its lowest energy triplet metal-to-ligand charge transfer $\left({ }^{3}\right.$ MLCT $)$ excited state. The quantum yield for the formation of this state is near unity $(11,12)$, implying that relaxation in this molecule is dominated by intersystem crossing from the ${ }^{1} \mathrm{MLCT}$ to the ${ }^{3} \mathrm{MLCT}$ manifold instead of "spin-allowed" processes associated with internal conversion or fluorescence. Despite some arguments for a delocalized excited state (13-15), various absorption (16-18), electroabsorption (19), and Raman experiments $(20-22)$ indicate an excited state with charge localized on a single ligand. In addition, polarized absorption $(23,24)$ and two-color Raman (25) experiments have revealed dynamics attributed to interligand electron transfer, again implying charge localization of the long-lived ${ }^{3}$ MLCT excited state. Previous work from our laboratory fo- cused on time-resolving the formation of the ${ }^{3}$ MLCT excited state with transient absorption pump-probe spectroscopy (26). The differential absorption spectrum observed $\geq 300$ fs after initial excitation matched that acquired on the nanosecond time scale, an indication that the formation of the ${ }^{3}$ MLCT state occurs with a time constant of $\tau \sim 100$ fs. Although this study defined the overall time scale for excited-state formation, it left unanswered a number of important, fundamental questions concerning the nature of the excited state (such as whether it is localized or delocalized) and the role of the solvent in the evolution of the charge transfer state. Here, we address both of these issues by examining the time dependence of the anisotropic absorption of the molecule.

The principal axis of symmetry in $\left[\mathrm{Ru}(\mathrm{bpy})_{3}\right]^{2+}$ is three-fold orthonormal to the plane bisecting all three bipyridine ligands. The ligands themselves are each bisected by a $\mathrm{C}_{2}$ axis (Scheme 1), thus rendering all three bipyri-

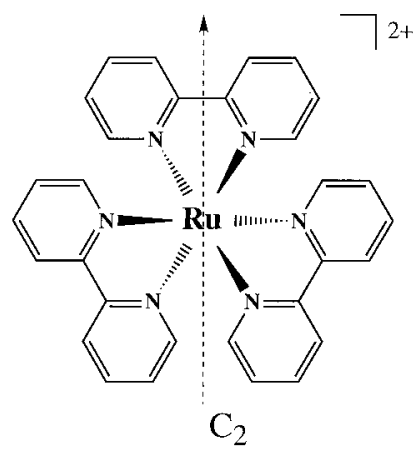

Scheme 1.

dine ligands indistinguishable in the ground state. Upon photoexcitation, in which an electron is promoted from the metal to the ligand(s), the excited-state configurations that can arise are indicated in Scheme 2. In the case of intrinsic delocalization (case a), the transition moment will be doubly degenerate with $(x, y)$ polarization, and the ligands remain indistinguishable. In contrast, if the electron is localized on a single bipyridine ligand, the degeneracy of the transition moment is lifted concomitant with the reduction in molecular symmetry from $\mathrm{D}_{3}$ to $\mathrm{C}_{2}$. This situation can be realized in the initially formed excited state (case b) or may occur as the result of dynamics from a delocalized configuration (case c). A time-resolved anisotropy

Scheme 2

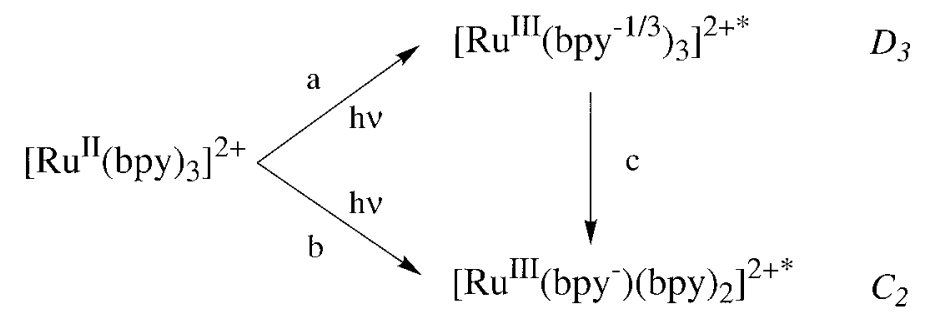

$C_{2}$ 


\section{R E P O R T S}

measurement can, in principle, distinguish among these possibilities.

The theory for anisotropy experiments is well developed for fluorescence spectroscopy (27) and is the basis for understanding the analogous transient differential absorption (i.e., pump-probe) measurement. In general, the anisotropy is defined as the normalized difference between the intensity of light (fluorescence or probe) polarized parallel $\left(I_{\|}\right)$and perpendicular $\left(I_{\perp}\right)$ to the excitation polarization,

$$
r=\frac{I_{\|}-I_{\perp}}{I_{\|}+2 I_{\perp}}
$$

In an isotropic distribution of nondegenerate transition dipole moments $(\mu)$,

$$
\begin{aligned}
I_{\|}-I_{\perp} & =\frac{1}{15} \int_{0}^{\infty}\left\langle 3[\mu(0) \mu(t)]^{2}\right. \\
& \left.-\mu^{2}(0) \mu^{2}(t)\right) \exp \left(-\frac{t}{\tau}\right) d t
\end{aligned}
$$

is the anisotropic term, dependent on the relative orientations of the transition moment normalized by system being probed at a time $t$ and the transition moment that was initially excited. This is

$I_{\|}+2 I_{\perp}=\frac{1}{3} \int_{0}^{\infty}\left\langle\mu^{2}(0) \mu^{2}(t)\right\rangle \exp \left(-\frac{t}{\tau}\right) d t$

which has no polarization dependence and thus represents the population kinetics of the system. The overall anisotropy of the

$r=\frac{2}{5} \int_{0}^{\infty}\left\langle P_{2}[\mu(0) \mu(t)]\right\rangle \exp \left(-\frac{t}{\tau}\right) d\left(\frac{t}{\tau}\right)$

follows a second-order Legendre polynomial, $P_{2}(\cos \theta)$, and has a maximum value of $r=0.4$. The situation becomes more complex in a pump-probe measurement because of potential contributions to the transient spectrum from excited-state absorption(s) and/or stimulated emission. The total observed anisotropy at the probe wavelength, $r$, is then given by

Fig. 1. Simultaneously acquired pump-probe scans of $\left[\mathrm{Ru}(\mathrm{bPy})_{3}\right]^{2+}$ at $480 \mathrm{~nm}$ in $\mathrm{CH}_{3} \mathrm{CN}$ with relative pumpprobe polarizations as indicated. The magic angle $\left(54.7^{\circ}\right)$ pumpprobe scan was scaled for inclusion in the figure; these data are insensitive to anisotropic effects and thus reflect population dynamics observable at this probe wavelength.

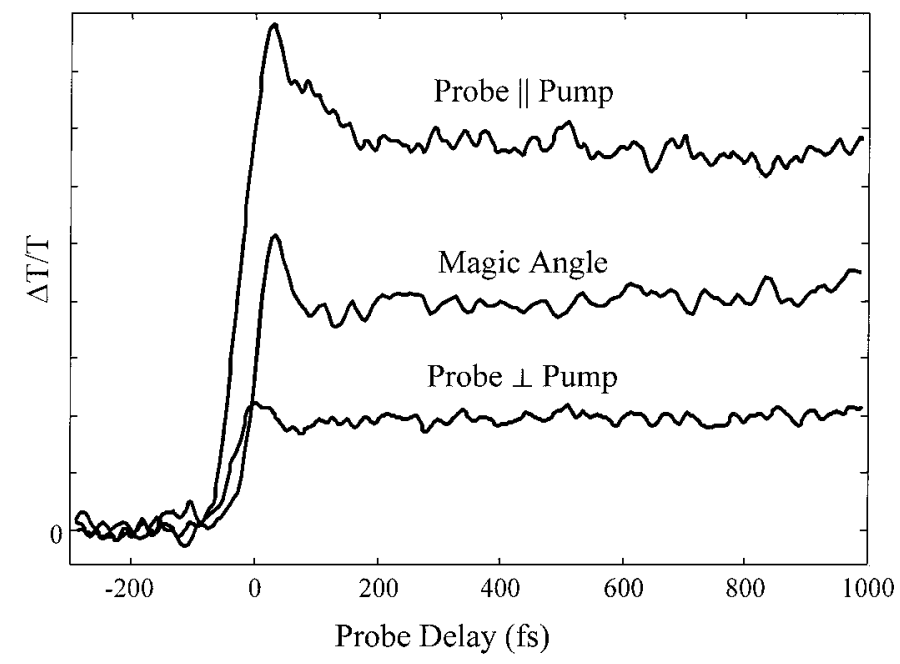

Fig. 2. Solvent and time dependence of the anisotropic absorption of $\left[\mathrm{Ru}(\mathrm{bPy})_{3}\right]^{2+}$ in $\mathrm{CH}_{3} \mathrm{CN}(\mathbf{A}), \mathrm{CH}_{3} \mathrm{CH}_{2} \mathrm{CN}$ (B), and $\mathrm{CH}_{3} \mathrm{CH}_{2} \mathrm{CH}_{2} \mathrm{CN}$ (C).

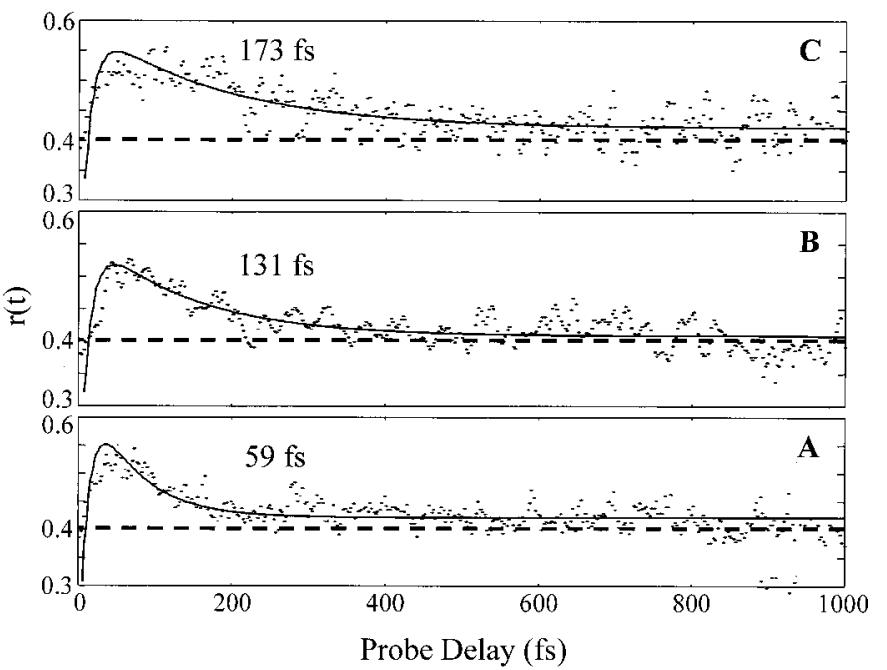

$$
r=[\alpha /(\alpha+\beta+\gamma)] r_{\alpha}+[\beta /(\alpha+\beta+\gamma)] r_{\beta}+
$$$$
[\gamma /(\alpha+\beta+\gamma)] r_{\gamma}
$$

where the values for $r_{\mathrm{i}}$ reflect the degeneracy and relative orientation of the transition dipole of each component and $\alpha, \beta$, and $\gamma$ are coefficients weighting the contributions from ground-state bleach, excited-state absorption, and stimulated emission, respectively (28).

The generation of optical pulses used in the ultrafast experiments has been described elsewhere (29). For these anisotropy measurements on $\left[\mathrm{Ru}(\mathrm{bpy})_{3}\right]^{2+}(30)$, the center wavelength of the pump and probe pulses was $480 \mathrm{~nm}$. This wavelength is at the red edge of the ${ }^{1}$ MLCT ground-state absorption envelope; density functional calculations show this region to be dominated by a doubly degenerate, $(x, y)$-polarized absorption (31). Figure 1 shows simultaneously acquired pumpprobe traces of $\left[\mathrm{Ru}(\mathrm{bpy})_{3}\right]^{2+}$ in $\mathrm{CH}_{3} \mathrm{CN}$ with the probe polarization parallel and perpendicular to the pump polarization along with data acquired at the magic angle. It is evident that there are anisotropic contributions to the parallel and perpendicular traces that cannot be attributed to population dynamics. The parallel and perpendicular traces in $\mathrm{CH}_{3} \mathrm{CN}$ and similarly collected data in $\mathrm{CH}_{3} \mathrm{CH}_{2} \mathrm{CN}$ and $\mathrm{CH}_{3} \mathrm{CH}_{2} \mathrm{CH}_{2} \mathrm{CN}$ were used to construct the time-dependent anisotropy traces (Fig. 2). The time-dependent absorption anisotropy of $\left[\mathrm{Ru}(\mathrm{bpy})_{3}\right]^{2+}$ in all three solvents has an initial value of $r \sim 0.55$ that depolarizes to $r \sim 0.4$; this latter value persists out to at least 1 ps $(32,33)$.

At $480 \mathrm{~nm}, \sim 35 \%$ of the differential absorption signal arises from absorption(s) of the reduced bpy polarized parallel to the long conjugation axis of the ligand (parallel to the 2,2' carbon-carbon bond) $(34,35)$. Thus, $\alpha=0.65$ and $\beta=-0.35$, yielding a value of $r=0.45$ in $\mathrm{C}_{2}$ symmetry (36). The experimental value of $r$ at long delay times is therefore consistent with charge localization on a single ligand and a formulation of $\left[\mathrm{Ru}\left(\mathrm{bpy}^{-}\right)(\mathrm{bpy})_{2}\right]^{2+}$ for the excited state sampled subsequent to the observed dynamics. The time-dependent nature of $r$ at early times rules out simple static localization (37), whereas depolarization due to interligand electron transfer has been assigned on the picosecond time scale. $(23,25)$. An initially localized excited state (case b) is therefore inconsistent with the anisotropic absorption data illustrated in Figs. 1 and 2.

For a delocalized state, the excited-state absorption dipole lies at the magic angle relative to the $(x, y)$ plane: $r_{\beta}$ is thus 0 and the differential absorption signal is due entirely to $r_{\alpha}$. Anisotropy values greater than $r_{\mathrm{i}}=0.4$ (Eq. 3) can only be seen in pump-probe measurements sampling transition moments of two-fold or higher degeneracy $(38,39)$. In such systems, the pump pulse creates a superposition of the degenerate excited states in 


\section{R E P O R T S}

the sample. Within the dephasing time $\left(T_{2}\right)$ of the system, the ratio of perpendicular to parallel pump-probe signals is equivalent to the Raman depolarization ratio: For a doubly degenerate transition moment, this anisotropy value is $r_{\mathrm{i}}=$ 0.7. Dephasing manifests itself as a reduction in the anisotropy. For a fully dephased, doubly degenerate system, the anisotropy of pump probe will be the same as in fluorescence, $r_{\mathrm{i}}=$ $0.1 \quad(38,39)$. Following excitation in $\left[\mathrm{Ru}(\mathrm{bpy})_{3}\right]^{2+}$ at $480 \mathrm{~nm}$, the observed anisotropy of the delocalized state can therefore range from 1.52 (fully coherent) to 0.22 (fully dephased). The latter value lies well below the asymptote of $r(t) \sim 0.4$ achieved in all three solvents, further supporting charge localization at long time delays. Given this and the initial values observed for $r(t)$, we conclude that our results are indicative of sampling a partially dephased (40) two-fold degenerate transition dipole at the earliest delay times followed by a reduction in symmetry to yield a nondegenerate state, dynamics conforming to case $\mathrm{c}$ in Scheme 2 (41).

As seen in Fig. 2, the rate of charge localization is observed to be dependent on solvent (see also Table 1). This solvent dependence can be attributed to the change in solvation that likely accompanies charge transfer. The nitrile solvents are polar and before excitation will solvate the dicationic $\left[\mathrm{Ru}(\mathrm{bpy})_{3}\right]^{2+}$ in such a way as to screen charge. Thus, the negative end of the solvent dipole (the $\mathrm{CN}$ group) will be oriented toward the solute in the ground state. This electrostatic condition is changed markedly with the introduction of additional negative charge density on the periphery of the chromophore in the MLCT excited state. The initial response to this inversion will most likely be dictated by nondiffusive motion of the solvent. The time constant of $\tau \sim 60$ fs observed for $\mathrm{CH}_{3} \mathrm{CN}$ is similar to values reported for the inertial dynamics of this solvent (42). Furthermore, it can be seen that the time scale for symmetry reduction in $\left[\mathrm{Ru}(\mathrm{bpy})_{3}\right]^{2+}$ across the solvent series is strongly correlated with the moment of inertia of the solvent molecule (Table 1). We therefore suggest that charge localization is coupled to and/or controlled by inertial solvation dynamics occurring in response to photo-induced charge transfer. The underlying driving force for charge localization is speculated to be coulombic, possibly as a means of minimizing the number of unfavorable solvent-solute interactions in the excited state.

Transient absorption measurements were carried out at the magic angle to assess the correlation between population dynamics and the time scale for charge localization (43, 44 ). As seen previously, the differential spectra evolve out to a delay of $\sim 300 \mathrm{fs}$, at which point the spectra match data acquired on the nanosecond time scale (Fig. 3A). A time constant of $\tau \sim 100$ fs can therefore be estimated for ${ }^{3}$ MLCT state formation. Although this differs from the 60 -fs depolarization time in this solvent, it is difficult to ascribe any importance to this difference because of the approximate nature of $\tau$ assigned to the spectral evolution. Time-resolved transient absorption data obtained for $\left[\mathrm{Ru}(\mathrm{bpy})_{3}\right]^{2+}$ in $\mathrm{CH}_{3} \mathrm{CH}_{2} \mathrm{CH}_{2} \mathrm{CN}$ are also shown (Fig. 3B). It can be seen that the rate of the spectral evolution in $\mathrm{CH}_{3} \mathrm{CH}_{2} \mathrm{CH}_{2} \mathrm{CN}$ occurs on a faster time scale than the corresponding change in anisotropy. Moreover, the population dynamics are essentially identical to those observed in $\mathrm{CH}_{3} \mathrm{CN}$ (Fig. 3A). We repeated this measurement in $\mathrm{CH}_{3} \mathrm{OH}$ and formamide solutions and obtained identical results in all cases. We therefore conclude that once sensitivity to anisotropy is removed from the experiment, the dynamics of excited-state evolution in the chromophore appear to be independent of solvent. This suggests that the wave packet motion associated with population dynamics is governed by intramolecular factors and occurs relatively independently of environmental forces. Whether the two processes we have identified are coupled is not yet clear.

Although specific, long-standing questions concerning the excited-state properties of $\left[\mathrm{Ru}(\mathrm{bpy})_{3}\right]^{2+}$ have been answered, the importance of the results presented herein lies in defining and differentiating solvent and intramolecular contributions to the evolution of

Table 1. Absorption depolarization dynamics of $\left[\mathrm{Ru}(\mathrm{bpy})_{3}\right]^{2+}$ in nitrile solution.

\begin{tabular}{lcccc}
\hline \multicolumn{1}{c}{ Solvent } & $\tau(\mathrm{fs})^{*}$ & $\tau_{\text {norm }} \dagger$ & $I\left(\mathrm{amu} \AA^{2}\right) \ddagger$ & $I_{\text {norm }}^{\dagger}$ \\
\hline $\mathrm{CH}_{3} \mathrm{CN}$ & 59 & 1.0 & 44.4 & 1.0 \\
$\mathrm{CH}_{3} \mathrm{CH}_{2} \mathrm{CN}$ & 131 & 2.2 & 78.6 & 1.8 \\
$\mathrm{CH}_{3}\left(\mathrm{CH}_{2}\right)_{2} \mathrm{CN}$ & 173 & 2.9 & 142 & 3.2 \\
\hline
\end{tabular}

*Time constants for fitting depolarization dynamics illustrated in Fig. 2 to single exponential decay models. Values for $r(0)$ were found to be similar $(0.60 \pm 0.05)$ for all three solvents. Error bars for the time constants are estimated to be $\pm 5 \%$ on the basis of analyses of multiple independent data sets. $\quad$ Normalized to $\mathrm{CH}_{3} \mathrm{CN}$. Inertial moment, calculated on the basis of the geometry of the molecule about its center of mass.

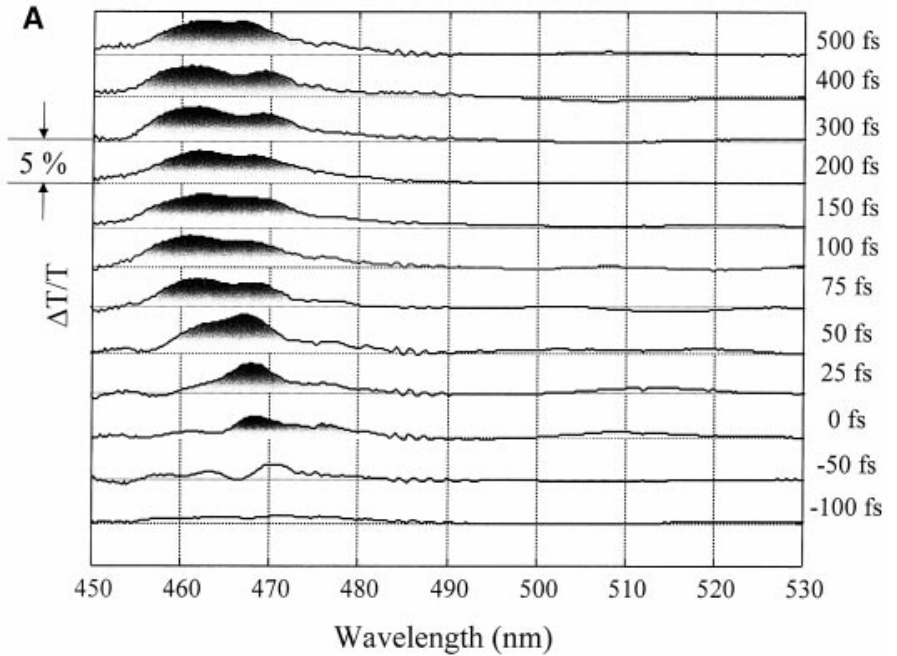

Fig. 3. Time-resolved transient absorption spectra, plotted as change in transmission $(\Delta T / T)$, for $\left[\mathrm{Ru}(\text { bpy })_{3}\right]^{2+}$ in $\mathrm{CH}_{3} \mathrm{CN}(\mathbf{A})$ and $\mathrm{CH}_{3} \mathrm{CH}_{2} \mathrm{CH}_{2} \mathrm{CN}$ (B). The spectra are offset by probe delay as labeled. A positive signal corresponds to an increase in the transmission of the probe, that is, bleach of the ground-state absorption. Data for $\Delta t \geq 300 \mathrm{fs}$ match

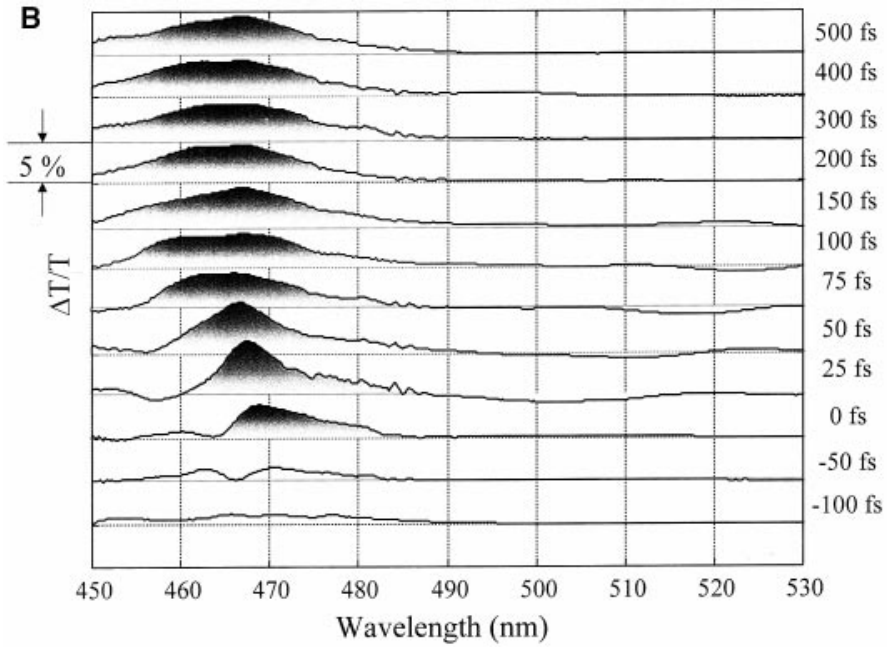

spectra obtained on the nanosecond time scale and therefore correspond to the ${ }^{3}$ MLCT state. Similar data were also obtained in $\mathrm{CH}_{3} \mathrm{OH}$ and formamide solution, indicating that the population dynamics sampled by these magic angle spectra are the same in all of these solvents. 
charge transfer states. In particular, it has been demonstrated that inertial solvation dynamics likely play a key role in the process of charge localization after photo-induced charge transfer. It is unclear to what extent these results can be extended to other, more complex chemical and biological systems. However, given the desirability for vectorial charge transport in various contexts, we believe that medium-induced localization dynamics may be important in a wide range of settings.

\section{References and Notes}

1. D. O. Hall and K. K. Rao, Eds., Photosynthesis (Cambridge Univ. Press, New York, ed. 5, 1995).

2. S. K. Deb, Curr. Opin. Solid State Mater. Sci. 3, 51 (1998).

3. G. A. Voth and R. M. Hochstrasser, J. Phys. Chem. 100, 13034 (1996).

4. R. M. Stratt and M. Maroncelli, J. Phys. Chem. 100, 12981 (1996).

5. M. Maroncelli, J. Mol. Liq. 57, 1 (1993).

6. G. R. Fleming and M. H. Cho, Annu. Rev. Phys. Chem. 47, 109 (1996).

7. C. J. Cramer and D. G. Truhlar, Chem. Rev. 99, 2161 (1999).

8. F. O. Raineri and H. L. Friedman, Adv. Chem. Phys. 107, 81 (1999).

9. V. Balzani, A. Juris, M. Venturi, S. Campagna, S. Serroni, Chem. Rev. 96, 759 (1996), and references therein.

10. K. Kalyanasundaram and M. Grätzel, Coord. Chem. Rev. 177, 347 (1998).

11. J. N. Demas and G. A. Crosby, J. Am. Chem. Soc. 93, 2841 (1971).

12. J. N. Demas and D. G. Taylor, Inorg. Chem. 18, 3177 (1979).

13. H. Yersin, W. Humbs, J. Strasser, Coord. Chem. Rev. 159, 325 (1997), and references therein.

14. D. Braun, E. Gallhuber, H. Yersin, Chem. Phys. Lett. 171, 122 (1990).

15. D. Braun, P. Huber, J. Wudy, J. Schmidt, H. Yersin, J. Phys. Chem. 98, 8044 (1994).

16. H. Riesen, L. Wallace, E. Krausz, Inorg. Chem. 35 6908 (1996).

17. J. S. Gold, S. J. Milder, J. W. Lewis, D. S. Kliger, J. Am. Chem. Soc. 107, 8285 (1985).

18. E. M. Kober, B. P. Sullivan, T. J. Meyer, Inorg. Chem. 23, 2098 (1984).

19. D. H. Oh, M. Sano, S. G. Boxer, J. Am. Chem. Soc. $\mathbf{1 1 3}$ 6880 (1991), and references therein.

20. P. J. Carroll and L. E. Brus, J. Am. Chem. Soc. 109, 7613 (1987).

21. P. G. Bradley, N. Kress, B. A. Hornberger, R. F. Dallinger, W. H. Woodruff, J. Am. Chem. Soc. 103, 7441 (1981).

22. M. Forster and R. E. Hester, Chem. Phys. Lett. 81, 42 (1981).

23. L. F. Cooley, P. Bergquist, D. F. Kelley, J. Am. Chem. Soc. 112, 2612 (1990)

24. R. A. Malone and D. F. Kelley, J. Chem. Phys. 95, 8970 (1991).

25. T. Yabe et al., J. Phys. Chem. 94, 7128 (1990).

26. N. H. Damrauer et al., Science 275, 54 (1997).

27. R. G. Gordon, J. Chem. Phys. 45, 1643 (1966).

28. D. M. Jonas, M. J. Lang, Y. Nagasawa, T. Joo, G. R. Fleming, J. Phys. Chem. 100, 12660 (1996).

29. R. W. Schoenlein, J.-Y. Bigot, M. T. Portella, C. V. Shank, Appl. Phys. Lett. 58, 801 (1991).

30. The pulses used for the time-resolved anisotropy measurements were $25 \mathrm{fs}$ in duration centered at 480 $\mathrm{nm}$ at a repetition rate of $540 \mathrm{~Hz}$. The polarization of the probe beam was oriented $45^{\circ}$ to the pump. After the beams were crossed in the sample, the probe was split by a polarization beam-splitting cube to facilitate simultaneous detection of polarization components parallel and perpendicular to the pump. The measured anisotropies were calibrated with control experiments on laser dye samples that yielded values of $r=0.4$ for nondegenerate transition dipoles.
31. C. Daul, E. J. Baerends, P. Vernooijs, Inorg. Chem. 33, 3538 (1994).

32. Values for $r(t)$ were found to be independent of probe wavelength in the range of 465 to $500 \mathrm{~nm}$ on the basis of anisotropy data reconstructed from polarized full spectra.

33. Rotational depolarization of this molecule occurs on the time scale of several tens of $p s$ in fluid solution [cf. (23)].

34. N. Sutin and C. Creutz, Adv. Chem. Ser. 168, 1 (1978); B. T. Weldon and J. K. McCusker, in preparation.

35. H. Hiratsuka, K. Sekiguchi, Y. Hatano, Y. Tanizaki, Y. Mori, Can. J. Chem. 65, 1185 (1987).

36. This calculation assumes random selection of the three possible MLCT transition dipoles; thus, $r_{\alpha}=0.1$. In addition, we do not observe stimulated emission from this compound $(\gamma=0)$.

37. Time dependence of the anisotropy arising from spectral shifting is considered to be unlikely because of the probe wavelength independence of $r(t)$ [cf. (32)].

38. C. Galli, K. Wynne, S. M. LeCours, M. J. Therien, R. M. Hochstrasser, Chem. Phys. Lett. 206, 493 (1993).

39. K. Wynne and R. M. Hochstrasser, Chem. Phys. 171, 179 (1993).

40. A value of $r(0)$ between 1.52 and 0.22 is to be expected if the electronic dephasing time is substantially (though not infinitely) shorter than the excitation pulse. Values of $r(0) \sim 0.6$ (Table 1) are therefore consistent with partial dephasing in $\left[\mathrm{Ru}(\mathrm{bpy})_{3}\right]^{2+}$ within the $\sim 25$-fs excitation pulse.

41. A similar model has been suggested by other workers on the basis of static variable-temperature emission spectra. See J. Ferguson and E. R. Krausz, J. Phys. Chem. 91, 3161 (1987), and references therein.

42. S. A. Passino, Y. Nagasawa, G. R. Fleming, J. Chem Phys. 107, 6094 (1997), and references therein.

43. A 25 -fs pump pulse centered at $480 \mathrm{~nm}$ and a $10-\mathrm{fs}$ broadband probe were used for the transient absorption measurements. After the pump and probe were crossed in the sample, the broadband probe was coupled into an optical multichannel analyzer for spectral measurements.

44. C. H. Brito Cruz, R. L. Fork, C. V. Shank, Opt. Lett. 12, 483 (1987).

45. We thank G. Fleming for helpful discussions. This research was supported by the Director, Office of Science, Office of Basic Energy Sciences of the U.S. Department of Energy grant DE-FG03-96ER14665 (J.K.M.) and contract DE-AC03-76F00098 (C.V.S.).

20 March 2000; accepted 14 June 2000

\section{$\mathrm{O}_{2}$ Activation by Nonheme Iron Complexes: A Monomeric Fe(III)-Oxo Complex Derived From $\mathrm{O}_{2}$}

\section{Cora E. MacBeth, ${ }^{1}$ Adina P. Golombek, ${ }^{3}$ Victor G. Young Jr., ${ }^{4}$ Cheng Yang, ${ }^{1}$ Krzysztof Kuczera, ${ }^{1,2}$ Michael P. Hendrich, ${ }^{3}$}

A. S. Borovik ${ }^{1 *}$

Iron species with terminal oxo ligands are implicated as key intermediates in several synthetic and biochemical catalytic cycles. However, there is a dearth of structural information regarding these types of complexes because their instability has precluded isolation under ambient conditions. The isolation and structural characterization of an iron(III) complex with a terminal oxo ligand, derived directly from dioxygen $\left(\mathrm{O}_{2}\right)$, is reported. A stable structure resulted from placing the oxoiron unit within a synthetic cavity lined with hydrogenbonding groups. The cavity creates a microenvironment around the iron center that aids in regulating $\mathrm{O}_{2}$ activation and stabilizing the oxoiron unit. These cavities share properties with the active sites of metalloproteins, where function is correlated strongly with site structure.

The activation of $\mathrm{O}_{2}$ by $\mathrm{Fe}(\mathrm{II})$ complexes is of fundamental importance in biology. Heme and nonheme iron enzymes use $\mathrm{O}_{2}$ as the primary oxidant in various biochemical transformations (1-3). In many of these enzymes, iron species with terminal oxo ligands are proposed as key intermediates in the catalytic cycle. Spectroscopic and structural $(4,5)$ results are consistent with these species being high valent iron centers

1Department of Chemistry and ${ }^{2}$ Department of Molecular Biosciences, University of Kansas, Lawrence, KS 66045, USA. ${ }^{3}$ Department of Chemistry, CarnegieMellon University, Pittsburgh, PA 15213 , USA. ${ }^{4}$ Department of Chemistry, University of Minnesota, Minneapolis, MN 55455, USA.

*To whom correspondence should be addressed. having formal oxidation states of $\geq 4+$. In synthetic porphyrin systems, spectroscopic and mechanistic evidence suggests that these oxoiron species can be generated at temperatures $<-40^{\circ} \mathrm{C}(6)$, but these complexes have yet to be structurally characterized by $\mathrm{x}$-ray diffraction (XRD) methods. There is considerably less information on synthetic nonheme iron species with terminal oxo ligands - their existence is often inferred from mechanistic considerations alone $(7,8)$. Efforts to model mononuclear heme and nonheme oxoiron species derived from $\mathrm{O}_{2}$ are hindered by the strong thermodynamic preference of Fe(III) complexes to form $\mu$-oxo bridged diiron spe-

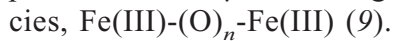

Metalloproteins overcome these obstacles 